
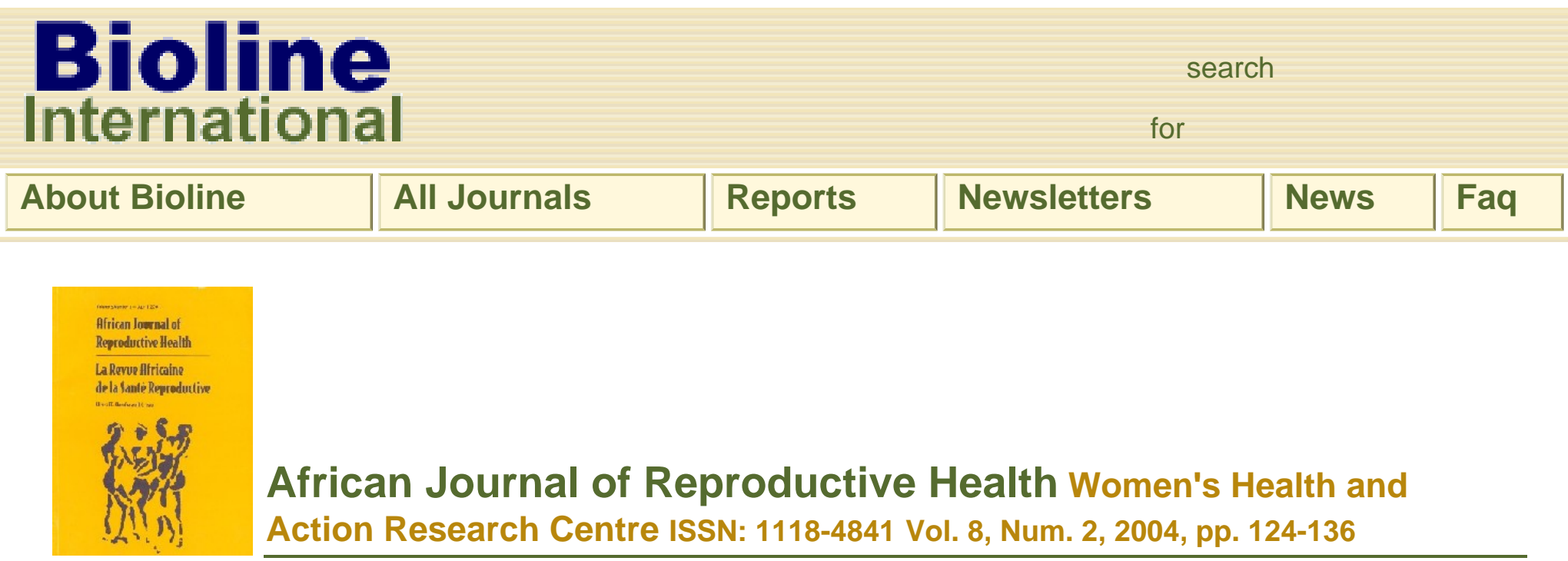

African Journal of Reproductive Health Women's Health and Action Research Centre ISSN: 1118-4841 Vol. 8, Num. 2, 2004, pp. 124-136

African Journal of Reproductive Health, Vol. 8, No. 2, Aug, 2004 pp. 124-136

\title{
Who is being Served Least by Family Planning Providers? A Study of Modern Contraceptive Use in Ghana, Tanzania and Zimbabwe
}

Steve Clements ${ }^{1}$ and Nyovani Madise ${ }^{2}$

1Steve Clements, Research Fellow, Opportunities and Choices Reproductive Health Knowledge Programme, Southampton Statistical Sciences Research Institute, University of Southampton, Southampton SO17 1BJ, United Kingdom.

E-mail: src@soton.ac.uk ${ }^{2} N y o v a n i$ Madise, Director, Centre for AIDS Research, School of Social Sciences, University of Southampton, Southampton SO17 1BJ, United Kingdom.

\section{Code Number: rh04034}

\section{Abstract}

This study was conducted to identify the poorest and other vulnerable sub-groups being served least by family planning providers. The study was set in three countries in sub-Saharan Africa, namely, Ghana, Tanzania and Zimbabwe. This region generally has a low but increasing uptake of modern contraceptive methods. As the use of family planning providers increases, there is a need to understand who is not being served and why. Logistic regression analyses of demographic and health survey data were conducted to identify the characteristics and geographical areas of women who are not using modern contraceptive methods. The results show some similarities among the countries in those using modern methods the least. However, a number of groups were country specific. Identifying the poorest women with the lowest use of modern methods is best done by assessing their household amenities or their partner's status rather than theirs. (Afr J Reprod Health 2004; 8[2]: 124-136 )

Key Words: Contraceptive use, sub-Saharan Africa, poverty 


\section{Résumé}

Qui sont les moins servis par les prestataires des services le la planification familiale? Une étude de l'usage moderne du contraceptif au Ghana, en Tanzanie et au Zimbabwe. Cette étude avait pour but d'identifier les groupes les plus pauvres et les autres sous-groupes qui sont les moins servis par les prestataires des services de la planification familiale. L'étude s'est située dans trois pays sub-sahariens, à savoir le Ghana, la Tanzanie et le Zimbabwe. Cette région connaît en général une faible acceptation des méthodes contraceptives modernes quoique cette acceptation soit en augmentation. Au fur et à mesure que le nombre des prestations des services de planification familiale augmente, il y a le besoin des comprendre ceux qui ne sont pas servis et pourquoi. Les analyses de la regression logistique des données de l'enquête démographique et de santé ont été menées pour identifier les caractéristitques et les domaines géographiques des femmes qui n'emploient pas des méthodes contraceptives modernes. Les résultats ont montré des similarités parmi les pays chez ceux qui emploient les méthodes modernes. Néanmoins, certains groupes affichaient des caractéristiques des pays spécifiques. Pour identifier les femmes les plus pauvres qui emploient les moins les méthodes modernes, il vaut mieux évaluer les appareils ménagers ou la situation économique de leur conjoint plutôt que de leur proper situation. (Rev Afr Santé Reprod 2004; 8[2]: 124-136)

\section{Introduction}

The use of family planning services is an important issue for the developing world, because of the benefits gained in terms of development, through reducing fertility levels. Uptake of family planning widens choices available to people, particularly women, by allowing individuals and, thus, society more opportunity for social and economic development. ${ }^{1}$ The poorest are often those with the least contraceptive use and the highest levels of fertility, and thus are most restricted in their ability to develop other activities. Furthermore, limited economic development tends to restrict social development, for example, in areas such as women's rights and equality. ${ }^{2}$ Thus, those women living in the poorest socio-economic circum-stances are also more likely to have to conform to traditional values or men's opinions regarding contraceptive use and childbearing. Consequ-ently, there may exist high poverty, low contraception and high fertility cycle that needs to be broken.

The key aim of this study is to identify those sub-groups who are currently under-served by providers for three countries in sub-Saharan Africa (Ghana, Tanzania and Zimbabwe) through secondary analysis of demographic and health surveys (DHS) data. This region generally has a low uptake of modern contraceptive methods and rapid rates of population growth, particularly in poorer urban areas. Common themes and disparities between the findings for each country are identified, as are the most appropriate indicators of poverty.

Initially this study explores which low users groups are identified by alternative measures of family planning need. Specifically, how modern contraceptive use, unmet need and intention to use contraception varies by demographic, residential and socio-economic factors. It is then outlined, in this case, why modern contraceptive use is the most appropriate indicator to 
analyse. Finally, the factors most associated with current use of a modern contraceptive method in each country are identified using statistical modelling techniques.

As a key objective of the study is to examine who is and who is not using contraception, it is necessary to analyse countries with a reasonable level of contraceptive prevalence so that such variations exist. Given the generally low levels of modern contraceptive use in sub-Saharan Africa, it was decided that in the countries selected current use of any modern contraceptive method by all women should be at least $10 \%$. The three countries, Ghana, Tanzania and Zimbabwe, were selected on this basis. Thus, geographically speaking this study covers one country from each of East, West and Southern Africa.

Table 1 illustrates that the percentage of all women of reproductive age (15-49 years) currently using a modern contraceptive method is much higher in Zimbabwe (31\%) than the other two countries. Zimbabwe is also the wealthiest of the three countries, and it has the lowest total fertility rate (TFR). In recognition of the importance of family planning, in 1985 the Zimbabwe National Family Planning Council became a state agency. Along with other government run health facilities, it is now the primary source of modern contraceptive methods. Communitybased distribution is also an important source. ${ }^{3}$

Ghana first adopted a population policy in 1969, which was revised in 1994. It specifically aims to reduce the total fertility rate to three by 2020 , partly through increasing the contraceptive prevalence rate to $50 \% .{ }^{4}$ In 1998 , Ghana had a slightly lower use of modern contraception than Tanzania. However, it has a markedly lower TFR and it is a wealthier nation than Tanzania (Table 1). In Ghana, modern contraception is primarily provided through government run health facilities or family planning clinics and at private drug stores. 4 Tanzania adopted a population policy in 1992 with an emphasis on reducing population growth. There is a comprehensive network of government run health facilities across the country and modern contraceptive users primarily obtain contraceptives from these facilities. 5

\section{Measures of Contraceptive Need}

There are a number of ways of identifying those groups who are served least by family planning providers. The simplest and most straightforward measure is the current use of modern contrace-ptive methods. Other measures include unmet need, intention to use contraception by non-users and unintended pregnancies by users. The unmet need and intention to use measures both identify different types of need. The intention to use measure simply identifies those current non-users who wish to use contraception in the near future. Unmet need is a more complex measure that uses information on fertility preferences and current contraceptive use to identify those who wish to regulate their fertility and, therefore, require contraception.

The concept and measurement of unmet need is open to debate. Essentially, it is the gap between the desire to avoid pregnancy and the failure to use contraception. ${ }^{6}$ It can also be subdivided into unmet need for limiting and unmet need for spacing. Unmet need can vary depending on whether all methods or only modern methods of contraception are taken into 
consideration and exactly which women are considered at risk of becoming pregnant. Typically, both the unmet need and intention to use measures tend to be based upon all methods of contraception. This is constrained by the questionnaire design of the DHS studies.

In general, most measurements of unmet need, and other contraceptive demand measures, are taken from cross-sectional surveys. These will vary from time to time, independent of contraceptive provision changes because of demographic, socio-economic and cultural factors. Furthermore, the questions used to calculate unmet need are argued by some to be inappropriate for the lifestyles of women in the developing world, where planning parenthood and understanding the costs of childbearing are not customary. ${ }^{1}$ Peruvian research has suggested that the focus of family planning programmes should be targeted at preventing unintended pregnancies amongst current users rather than persuading non-users to become users. $^{7}$

Overall, the circumstances of the country, such as the level of contraceptive prevalence and family planning policy, or the objectives of the study, may dictate which measurement of need is best to focus upon. Furthermore, as each measure tells a slightly different story, it may be better to examine a range of measures when considering the need for contraception in a particular country. Those intending to use contraception are obviously the easier to target, whilst those with unmet need are an important group of clients for family planning providers. Meanwhile, the poorest and other vulnerable groups may use modern methods the least because they least require them or want to use them.

For this study, it was decided to explore the relationship between various socio-demographic groups and modern contraceptive use, unmet need and intention to use contraception. However, for the final statistical analyses modern contraceptive use was chosen as the key measure of interest. This was because, in this case, the aim of the study is to identify the poorest and other sub-groups who are being served least by contraceptive providers. Consequently, it is natural to use a measure based solely on modern methods and one that is also strongly associated with poorer socio-economic groups. Such groups are likely to be "harder to reach" for family planning providers in terms of demand for contraception. However, it is argued that there are many benefits for the women concerned and developing countries in general in getting such groups to use contraception.

\section{Which Groups Use Contraception the Least?}

There are a large number of papers and surveys that have identified groups of women who use any contraceptive method less than others, though fewer have analysed the use of modern methods especially in sub-Saharan Africa. The compelling evidence is that those women in poor socio-economic circumstances or whose partners are in similar circumstances are less likely to use contraception. 8,9 For example, in some parts of sub-Saharan Africa women who have attended secondary education are ten times more likely to use contraception than those with no education. ${ }^{9}$ Education is typically used as a measure of socio-economic status, though it has been shown to effect fertility independent of socio-economic status. ${ }^{10}$ Other socio-economic indicators are based upon income, expenditure, occupation, household assets or access to 
basic amenities (water, sanitation, etc). However, there is no agreed appropriate way of defining the poorest from survey data because poverty is much more multi-dimensional than these measures suggest. ${ }^{11}$

Across the developing world there are other groups who have also been particularly identified as low contraceptive users. Broadly speaking, these include teenagers, unmarried women, those of a particular religious or traditional belief or from a particular ethnic group, those living in rural areas, specific regions of a country and refugees or displaced persons. ${ }^{8,9,12}$ Evidence for the use of modern methods across countries in sub-Saharan Africa generally confirms that the same sub-groups also use modern methods less. ${ }^{13}$

There is an obvious overlap between the sub-groups identified. For example, a lot of teenagers are likely to be living in the poorest urban areas. ${ }^{14}$ Thus, individuals may appear in more than one group and such a combination of characteristics could lead to even lower contraceptive use. ${ }^{8}$ Furthermore, the variation in the use of contraception by age and marital status is clearly linked to the level of sexual activity an individual experiences. Clearly, lower use of modern contraceptive methods by unmarried women could be because fewer of them have sexual partners as compared to married women.

The relationship between groups with certain characteristics and their use of contraception could be partially explained by cultural or service provision factors. It is often highlighted that the poorest of the poor and other vulnerable sub-groups have poor knowledge of, and limited access to, comprehensive family planning services of good quality. $6,8,15$ The environment within which the poorest and other vulnerable groups live, such as slum areas, may restrict the provision of services to them. Thus, better services are located in alternative areas that may be difficult for the women concerned to access. In general, family planning programmes can vary markedly between countries and within countries, and this may account for stronger associations between certain groups and their contraceptive use across different settings.

In each of the three countries analysed in this study, research examining fertility and contraceptive use has highlighted sub-groups of low contraceptive users. However, there has been limited detailed analysis of modern contraceptive use in the three countries analysed in this paper. This is probably because, apart from Zimbabwe, modern contraceptive use has typically been very low and any uptake has only occurred recently.

Furthermore, the factors considered in such studies tend to vary and they tend to include all women irrespective of their sexual activity. Evidence tends to indicate that urban-rural residence and socio-economic factors are the key correlates of modern contraceptive use in these countries. For Zimbabwe, women who are aged over 35 years, are less educated, have less household items/family assets and live in rural areas are less likely to use modern methods. 16,17 In Ghana, limited education, low household expenditure and being born in a rural area have been linked with low use of modern methods. ${ }^{18}$

In Tanzania, some research has examined issues relating to factors that may encourage or 
discourage the use of family planning services. In Dar-es-Salaam it has been found that appropriate services are not available for teenage women. This group were also not aware of their right to use existing family planning services. ${ }^{19}$ In rural areas, community level perceptions of family planning services influence contraceptive use. ${ }^{20}$ Elsewhere, the success of media campaigns have been linked with the increased use of modern methods. ${ }^{21,22}$

\section{Data and Methods}

The data used in this study were obtained from demographic and health surveys in each country. ${ }^{3-5}$ Table 2 displays details of the study population in each survey. This includes a conservative measurement of the number of women considered 'at risk' of conceiving in each survey and, therefore, potential contraceptive users. This was calculated by excluding, from all women interviewed, those women who were currently pregnant or who had not had previous sexual intercourse. This 'at-risk' population was used in all analyses for this study.

For purposes of the exploratory analysis, three measures of contraceptive use or need were calculated, namely, use of a modern method, unmet need, and future intentions to use contraception by non-users. Modern contraceptive use was defined as the proportion of 'at-risk' women currently using a modern method. Unmet need was defined using the DHS second definition typically used in their final reports. ${ }^{a}$ The intention to use contraception was defined as those at-risk women who were not currently using contraception but who were intending to use a method of contraception in the next 12 months.

a In this paper the following definition of unmet need is used: Pregnant women whose pregnancies were mistimed, postpartum amenorrheic women whose last births were mistimed, and fecund women who are neither pregnant nor postpartum amenorrheic and who are not using any method of family planning and say they want to wait two or more years for their next birth, are undecided about the timing of the next birth, or are undecided whether to have another child $1 / 4$ Pregnant women whose pregnancies were unwanted, postpartum amenorrheic women whose last birth was unwanted and fecund women who are neither pregnant nor postpartum amenorrheic and who are not using any method of family planning and who want no more children $3-5$

A number of background individual demographic, residential and socio-economic variables were available from the DHS datasets for analysis. bc The selection of these variables enables some identification of the groups who are and who are not being served by family planning providers. Individual knowledge, attitude and behaviour factors are not considered in this analysis. This is because the aim is to identify the background characteristics of those who use contraception the least and the regions of low use. However, whether the woman wants another child in the next 12 months is included in the analysis of contraceptive use. This is typically a strong predictor of use that is necessary to control for when analysing factors predicting contraceptive use.

${ }^{b}$ Variables tested in the models: Wants child in next 12 months; age; number of living children; current marital status; religion; ethnicity (Ghana); type of place of residence (urban / rural); 
region; education level; occupation; household amenities score; enough to eat (Tanzania); partner's educational level; partner's occupation.

c The marital status of the respondents was recoded to include polygynous unions, for both those women currently married and currently living together. This was calculated using the response to a question on the number of other wives that she shares her husband/partner with. In a few cases there was a missing response to this question, these women were grouped with those in monogamous relationships.

The study aims to be consistent in the factors used and the categories that factors are grouped into between the countries. However, there is some variation. This is to take account of the different distributions within each factor by country and country-specific factors, e.g., religious groups. Ethnic group is only included in the analysis for Ghana. There were too many ethnic groups for a sensible analysis in Tanzania, whilst in Zimbabwe ethnic group was not recorded in any detail. The DHS data include a number of indicators of household amenities. Owing to this and in order to restrict the number of variables in the final analysis, a household amenities score was calculated for each woman. ${ }^{d}$

d Seven variables were included in the household amenities score: whether her house had electricity; radio ownership; television ownership; fridge ownership; source of drinking water; toilet facilities and the type of floor material. The first four variables are all binary and were coded zero and one, with one indicating the presence of the amenity. The last three factors are categorical, these were grouped into three categories so that a limited amenity was given a zero score, a moderate amenity was given a score of a half, and those with a good amenity were given a score of one. A household amenity score was created by summing the scores of the seven variables. This was grouped into three categories (low, medium, high) for the purpose of the analysis, so that approximately a third of all women fell into each category. The specific cutoff points used were for Ghana: 0-2 (Low), 2.5-4 (Medium), 4.5+ (High); Tanzania: 0-1 (Low); 1.5-2 (Medium); 2.5+ (High); Zimbabwe: 0-1 (Low); 1.5-3 (Medium); 3.5+ (High).

Initially, an exploratory analysis was per-formed to examine how modern contraceptive use, unmet need and intention to use contraception were related to background demographic, residential and socio-economic factors in each of the three countries. It was noted earlier that modern contraceptive use appears to be the most appropriate measure to analyse for the purpose of this study. Thus, data for the use of a modern contraceptive method were modelled using survey regression techniques in the statistical package STATA. These regression techniques account for the design of the study when analysing the data. Specifically, the unequal sampling probabilities (weights), the strata and the primary sampling unit were controlled for by STATA. Thus, parameter estimates and their standard errors properly account for the design of each survey. ${ }^{23}$ The outcome variable, contra-ceptive use, was binary (use $=1$ ). Consequently, logistic survey regression methods were used to analyse the data.

A sequential model selection process was used to fit the data. Initially, whether the woman wants another child in the next 12 months was entered into the model. Then demographic, residential (urban-rural/region) and socio-economic factors were added to the model in turn. At 
each stage, forward selection and backward elimination techniques were used to identify significant factors (at the $5 \%$ level). All interactions were tested for. Once the final model was obtained a composite deprivation score was calculated.e This was to test whether any multiple deprivation effects existed apart from those already observed.

\section{Results}

\section{Exploratory Analysis}

Table 3 presents, for certain key factors, the percentage of 'at-risk' women who use modern methods, have unmet need for contraception, or intend to use contraception in the next 12 months in each country. In terms of socio-economic status (education and household amenities) it is clear that the poorer the women the less likely they are to be using a modern method. Generally, this is also the case with unmet need. However, the relationship isn't as strong and those from the lower to middle socio-economic groups can have similarly high levels of unmet need. Results for intention to use contraception are more mixed but tend to indicate a greater intention to use by those women from middle to higher socio-economic backgrounds.

The correlation between the measures across all factors in each country is given in Table 4 . In all three countries there was limited agreement between the factors most associated with low contraceptive use, high unmet need and high intention to use contraception. The best agreement was typically a negative correlation between contraceptive use and unmet need especially in Zimbabwe where contraceptive use is the greatest. Such results are to be expected as they illustrate who is being served in different ways. The unmet need and intention to use measures are also based on all methods of contra-ception.

\section{Regression Analyses}

Results from the survey logistic regression analyses of the use of a modern contraceptive method by women 'at-risk' of conceiving are presented in Table 5.

\section{Demographic Findings}

There were significant differences between modern contraceptive use for women of different reproductive ages. However, there was no consistent pattern between the countries. In Ghana, the results show that use of a modern method of contraception is lower for those aged over 39 years. In particular, women aged $45-49$ years had an OR 0.44 of using a modern method compared to those aged 30-39 years. Otherwise, modern contraceptive use was very similar in Ghana for those aged 15-39 years. In Tanzania, use of a modern method of contraception is lowest for those aged 15-19 years $(O R=0.61)$ as compared to those women aged 30-39 years. Otherwise no significant differences were found between any other age groups and women aged 30-39 years. In Zimbabwe, use of a modern method of contraception is lowest for those aged $45-49$ years $(O R=0.23)$ and $40-44$ years $(O R=0.57)$ as compared to women aged 30-39 years. Women aged 20-29 years are significantly more likely to be using a modern method. 
In general, modern contraceptive use increased with the number of living children a woman had. However, in Ghana, women with two living children were significantly less likely to use a modern method than those with no children $(\mathrm{OR}=0.59)$. In Tanzania and Zimbabwe, those with one or more children were all significantly more likely to use a modern method than those with no children.

Women who were formerly married tended to use modern contraception the least, whereas those in polygynous unions had a lower odds of use than those in monogamous unions. Modern contraceptive use for never married women was relatively high after other significant factors were controlled. In Ghana, women who were formerly married were much less likely to use contraception than those in polygynous $(O R=1.99)$ or monogamous unions $(O R=2.66)$. The never married were the most likely to be using a modern method (OR = 3.06). In Tanzania, never married women were more likely to being using a modern method than those of any other marital status. In Zimbabwe, women in monogamous unions (OR = 3.33) were the most likely to be using a modern method of contraception relative to formerly married women. Never married women and those in polygynous unions were also more likely to use modern contraception than the formerly married.

In all three countries, religion was also associated with modern method use. Those from traditional religions used modern contraception the least. In Ghana, women from traditional or other religions had the lowest use $(\mathrm{OR}=0.53)$ relative to Catholics. However, there is no significant difference in the use of modern methods by Protestants or Muslims relative to Catholics. In Tanzania, those of traditional religions have a much lower use of modern methods $(\mathrm{OR}=0.23)$ as compared to Catholics. Here, both Muslims $(\mathrm{OR}=1.31)$ and Protestants $(\mathrm{OR}=$ 1.56) used modern methods significantly more than Catholics. In Zimbabwe, Christians were significantly more likely to be using a modern contraceptive method than those of any other religion $(\mathrm{OR}=0.73)$.

\section{Residential Findings}

After accounting for socio-demographic factors, much regional variation still existed in modern contraceptive use in all countries. In Ghana, those living in the Volta, Eastern and the Upper East regions were significantly more likely to be using a modern method than those in the Western region, where the odds of modern contraceptive use was the least. In Tanzania, women living in the Lake zone used modern contraception the least. Otherwise, those living in the Coastal, Northern Highlands and Southern regional zones were significantly more likely to be using a modern method than those in the Lake zone. In Zimbabwe, women living in Bulawayo were the least likely to use modern methods. Otherwise, all other regions, except Matabeleland North and Manicaland, showed a significantly greater use of modern methods than Bulawayo. Furthermore, urban-rural residence was also a significant predictor of use except in Ghana. Women living in urban areas of Tanzania $(O R=1.78)$ and Zimbabwe $(O R=$ 1.61) were significantly more likely to use a modern method than those in rural areas. 
In general, a woman's educational level or occupation was not a strong predictor of modern contraceptive use. However, in Tanzania, modern contraceptive use did increase strongly with the educational level of a woman. Specifically, women with at least an incomplete primary education were more likely to use modern methods than those with no education. In Zimbabwe, unemployed women were the least likely to be using a modern method. All other occupations were significantly more likely to be using a modern method except skilled manual workers.

In all three countries women with the fewest household amenities were the least likely to be using a modern method of contraception. For example, in Ghana, those with high amenity (OR $=1.90)$ and medium amenity $(O R=1.64)$ scores were significantly more likely to be using a modern method than those women with low amenity score.

Partner's educational level and, in Tanzania partner's occupation, were significant predictors of modern contraceptive use. In Ghana, if the partner had received any form of education the woman was more likely to be using a modern method. However, there was no increase in the odds of use for higher educational levels of her partner. In Tanzania, women with partners who had completed primary education $(O R=1.71)$ and some secondary or higher education $(O R=$ 1.77) had a significantly higher use than those with no education. Those whose partners were self-employed in agriculture or unemployed were significantly less likely to be using a modern method than those in any other occupation $(O R=1.61)$. In Zimbabwe, only those with partners who had received secondary and higher education $(O R=1.78)$ were significantly more likely to be using a modern method than those with no education.

A composite socio-economic deprivation index of the significant socio-economic factors did not prove to be a further explanatory factor for modern contraceptive use in any country. This indicates that the combined effect of different socio-economic factors is additive and not multiplicative. As the only female-specific socio-economic indicator that remained significant in the final model was household amenities score, a composite socio-economic indicator was not tested in Ghana.

Women who desired more children in the next 12 months were less likely to be using a modern method. This group was included in the final data sets as they were at risk of conceiving and, if they did not want another child immediately, they were also potential contraceptive users.

Furthermore, it is debateable how much planning of children occurs in less developed societies, and if it does how much of this it is within the individual control of women. However, to check the validity of the findings, these women were removed from each of the final models. There was little change in the parameter values except for Ghana where women with one living child were significantly less likely to use modern methods $(\mathrm{OR}=0.58)$.

\section{Discussion and Conclusions}

The aim of this study was to identify the poorest and most vulnerable groups being served least by contraceptive providers. A number of measures of contraceptive demand may be appropriate to use in such a study, all of which are important when considering the wider contraceptive use in a country. However, we decided that the unmet need and intention to use measures were not 
appropriate because of the desire to identify sub-groups who use modern contraception the least and who may be the hardest to reach. These measures are not solely based on modern methods and findings from the exploratory analysis confirmed that modern contraceptive use is the measure most strongly associated with poorer socio-economic groups.

Three countries were analysed to identify those who were using modern contraceptive methods the least in each setting. However, it is clearly of interest to examine how these findings relate to each other and to the evidence found in other research. Although any interpretations are limited by using only three countries, some important themes stand out in the findings.

There is mixed agreement between the three countries in the factors shown by the regression models to be predictors of the low use of modern contraceptive methods. A number of socioeconomic factors are important. In particular, the woman's household amenity score was an important predictor in all three countries. The poorest, with fewer amenities, were less likely to be using a modern method. This may reflect higher opportunity costs of childbearing for wealthier women and higher product, consultation and transportation costs in obtaining contraception for poorer women. 8 This factor was also likely to be related to where women live, because those in urban areas were likely to have more household amenities.

Partner's educational level was also a significant socio-economic predictor of modern contraceptive use. Women whose partners had received no education were the least likely to be using modern methods. In some countries those with primary education were also less likely to use a modern method. This confirms the importance of education in empowering women to uptake contraception and challenge traditional childbearing norms. Furthermore, religion is also an important factor identifying those being served the least in all three countries. Specifically, those from traditional religions typically had the lowest use of modern contraception. This finding is consistent with evidence that within many traditional religions there are likely to be high fertility norms and constraints on women's autonomy. 24

Region of residence of a woman in a country was also an important factor predicting use of modern contraception, after accounting for demographic and socio-economic factors. This implies that other contextual factors such as service provision and infrastructure may be important in explaining the remaining regional variation. Furthermore, as highlighted in past research, there is some evidence that rural women are less likely to use modern methods, but again not in Ghana. This reflects social and economic underdevelopment in rural areas relative to urban areas. ${ }^{25}$

Some socio-economic indicators were not appropriate for identifying those being served the least. In particular, there was little evidence that a woman's educational level or occupation was associated with modern contraceptive use. The former was only significant in Tanzania and the latter only in Zimbabwe. Furthermore, in no country was a composite socio-economic index important. This indicates that for women with a combination of poor socio-economic factors, their chances of not using modern contraception are not multiplied. Instead, as no significant interactions were found, the effects of the socio-economic factors are additive. Contrary to other evidence, there is not a consistent relationship with women's age except for lower use by older 
women who were reaching the end of their reproductive life. The formerly married were low users in all three countries. This is perhaps because they were less likely to have a current sexual partner. Additionally, women in polygynous unions were less likely to use contraception than those in monogamous unions. However, contrary to previous literature, unmarried women tended to have a relatively high use of modern methods.

Clearly the choice of 'at-risk' women is important. Here, we have controlled for those who had never been sexually active, who were currently pregnant and those who desired a child in the next 12 months. There are different ways of doing this and how it is done will impact on the final results. This is especially so in the case of demographic factors such as age, marital status and parity, which are clearly related to sexual activity, pregnancy and the desire for children.

Exploratory analysis (Table 3 ) shows some evidence for the general consensus that teenagers and never married women use modern methods less. ${ }^{8,9,12}$ However, once other factors, including desire for children, were accounted for this was generally not the case (Table 5). Though age and marital status were still key determinants of modern contraceptive use, the least served sub- groups within them varied by country. It is typically women who may have been less sexually active (formerly married, 40+) and thus had fewer requirements for contraception that were served the least. In general, this implies that merely looking at age and marital status is not good for identifying vulnerable sub-groups of low contraceptive users.

Results for the socio-economic factors imply that identifying the poorest women with low modern contraceptive use appears to be best done by assessing their household amenities or partner's educational status rather than their own. This also highlights the limited independence of women, as their actions appear to depend upon their partner's and/or collective household socio-economic status. Otherwise, vulnerable sub-groups are country-specific. This is in terms of different traditional religions or places of residence.

In terms of further research, it is important to examine predicted contraceptive use in each cluster in the survey. This identifies outlying areas where contraceptive use may be especially high or low given its characteristics. Such areas are important to investigate in more depth to understand barriers or successful strategies that mark the relationship between use of modern methods and certain groups.

\section{Acknowledgements}

This paper was presented at the British Society for Population Studies Annual Conference, Leeds 3-5 September 2001. This research has been supported by the UK Government's Department for International Development. We are grateful for the permission granted by Measure DHS+ to use the relevant demographic and health surveys data sets.

\section{References}

1. World Bank. Family planning as a service. In: World Development Report. Chapter 7. 
Oxford: Oxford University Press, 1984.

2. Alan Guttmacher Institute. Hopes and Realities: Closing the Gap between Women's Aspirations and their Reproductive Experiences. New York: Alan Guttamacher Institute, 1998.

3. Central Statistical Office (Zimbabwe) and Macro International Inc. Zimbabwe Demographic and Health Survey, 1994. Calverton: Central Statistical Office and Macro International Inc., 1995.

4. Ghana Statistical Service (GSS) and Macro International Inc. (MI). Ghana Demographic and Health Survey 1998. Calverton: GSS and MI, 1999.

5. Bureau of Statistics (Tanzania) and Macro International Inc. Tanzania Demographic and Health Survey 1996. Calverton: Bureau of Statistics and Macro International, 1997.

6. Ross J, Stover J and Wilard A. Profiles for Family Planning and Reproductive Health Programs. Glastonbury: The Futures Group International, 2000.

7. Jain A. Should eliminating unmet need for contraception continue to be a program priority. Int Fam Plann Persp 1999; 25(Supplement): S39-S43, S49.

8. National Research Council. Factors Affecting Contraceptive Use in Sub-Saharan Africa. Washington DC: National Academy Press, 1993.

9. Curtis SL and Neitzel K. Contraceptive knowledge, use, and sources. Demographic and Health Surveys Comparative Studies No. 19. Calverton: Macro International Inc., 1996.

10. Cleland $\mathrm{J}$ and Rodriguez $\mathrm{G}$. The effect of parental education on marital fertility in developing countries. Pop Stud 1988; 42(3): 419-442.

11. Falkingham $\mathrm{J}$ and Namazie $\mathrm{C}$. Measuring health and poverty: a review of approaches to identifying the poor. London: DFID Health Systems Resource Centre, 2002.

12. Krause SK, Jones RK and Purdin SJ. Programmatic responses to refugees' reproductive health needs. Int Fam Plann Persp 2000; 26(4): 181-187.

13. Demographic and Health Surveys. Demographic and Health Surveys STATcompiler [online]. Measure DHS+, 2001. Available from: http://www.measuredhs.com

14. Mamdani M, Garner P, Harpham T and Campbell O. Fertility and contraceptive use in poor urban areas of developing countries. Health Pol Plann 1993; 8(1): 1-18.

15. Zulu EM, Ezeh AC and Dodoo FN. Slum Residence and Sexual Outcomes: Early Findings of Causal Linkages inNairobi, Kenya. Working Paper No.17. Nairobi: African Population and Health Research Center, 2000.

16. Thomas D and Maluccio J. Contraceptive Choice, Fertility and Public Policy in Zimbabwe. Living Standards Measurement Study Working Paper No. 109. Washington DC: World Bank, 1995.

17. Guilkey DK and Jayne S. Fertility transition in Zimbabwe: determinants of contraceptive use and method choice. Pop Stud 1997; 51(2): 173-189.

18. Oliver R. Contraceptive use in Ghana. The Role of Service Availability, Quality and Price. Living Standards Measurement Study Working Paper No. 111. Washington DC: World Bank, 1995. 
19. Rasch V, Silberschmidt M, Mchumvu Y and Mmary V. Adolescent girls with illegally induced abortion in Dar es Salaam: the discrepancy between sexual behaviour and lack of access to contraception. Reprod Health Matters 2000; 8(15): 52-62.

20. Mroz TA, Bollen KA, Speizer IS and Mancini DJ. Quality, accessibility, and contraceptive use in rural Tanzania. Demography 1999; 36(1): 23-40.

21. Jato MN, Simbakalia C, Tarasevich JM, Awasum DN, Kihinga CN and Ngirwamungu E. The impact of multimedia family planning promotion on contraceptive behavior of women in Tanzania. Int Fam Plann Persp 1999; 25(2): 60-67.

22. Rogers EM, Vaughan PW, Swalehe RM, Rao N, Svenkerud P and Sood S. Effects of an entertainment-education radio soap opera on family planning behavior in Tanzania. Stud Fam Plann 1999; 30(3): 193-211.

23. Madise N, Stephenson R, Matthews Z and Holmes D. Impact of estimation techniques on regression analyses: an application to survey data on child nutritional status in five African countries. Math Pop Stud (In press).

24. Caldwell JC and Caldwell P. The cultural context of high fertility in sub-Saharan Africa. Pop Dev Rev 1987; 13(3): 409-437.

25. Nazzar A, Adongo PB, Binka FN, Phillips JE and Debpuur C. Developing a culturally appropriate family planning program for the Navrongo experiment. Stud Fam Plann 1995; 26(6): 307-324.

(CWomen's Health and Action Research Centre 2004

\section{The following images related to this document are available:}

\section{Photo images}

[rh04034t4.jpg] [rh04034t1.jpg] [rh04034t3.jpg] [rh04034t2.jpg] [rh04034t5.jpg] 
Table 4 Spearman's Rank Correlation Coefficients Comparing Contraceptive Demand Measures

\begin{tabular}{lrrr}
\hline & $\begin{array}{r}\text { Modern contraceptive } \\
\text { use: unmet need }\end{array}$ & $\begin{array}{r}\text { Modern contraceptive } \\
\text { use: intention to use }\end{array}$ & $\begin{array}{r}\text { Unmet need: intention } \\
\text { to use }\end{array}$ \\
\hline Ghana & -0.31 & 0.20 & 0.34 \\
Tanzania & -0.37 & 0.68 & -0.21 \\
Zimbabwe & -0.57 & 0.20 & -0.16 \\
\hline
\end{tabular}




\section{Table 1 Profile of Study Countrics}

\begin{tabular}{lrrr}
\hline & Ghana & Tanzania & Zimbabwe \\
\hline Modern contraceptive use (\%) & 10.7 & 11.7 & 31.1 \\
Population (millions) & 18.9 & 30.6 & 11.0 \\
Total fertility ratc & 4.6 & 5.8 & 4.3 \\
GNP per capita (US\$) & 360 & 170 & 610 \\
Sounes: Contraceplive Use and TFR - Relenant DHS Reports; Population and GNP (Cross National Product) - Interna- \\
tional Planned Parenthood Federation (2001) Country Profiles.
\end{tabular}


Table 3 Measures of Female Contraceptive Demand by various Characteristics

\begin{tabular}{|c|c|c|c|c|c|c|c|c|c|}
\hline \multirow[t]{2}{*}{ Characteristic } & \multicolumn{3}{|c|}{ Modern contraceptive use } & \multicolumn{3}{|c|}{ Unmet need } & \multicolumn{3}{|c|}{ Intention to use } \\
\hline & Ghana & Tanzania & Zimbabwe & Ghana & Tanzania & Zimbabwe & Ghana & Tanzania & Zimbabwe \\
\hline 'At-risk' women & 13.6 & 15.2 & 44.0 & 21.1 & 19.8 & 10.5 & 25.6 & 33.0 & 45.3 \\
\hline Aged $15-19$ years & 13.4 & 7.4 & 31.9 & 20.5 & 22.2 & 11.4 & 30.6 & 38.8 & $\overline{624}$ \\
\hline Never married & 13.3 & 13.5 & 28.7 & 12.3 & 17.8 & 5.4 & 22.0 & 39.8 & 63.2 \\
\hline Rural residence & 12.0 & 10.8 & 39.2 & 23.0 & 20.8 & 12.1 & 26.4 & 31.0 & 45.2 \\
\hline No education & 9.2 & 5.5 & 29.1 & 23.5 & 18.5 & 13.2 & 22.1 & 20.0 & 30.2 \\
\hline $\begin{array}{l}\text { Some nrimary } \\
\text { education }\end{array}$ & 120 & 14.0 & 37.6 & 25.7 & 23.5 & 13.1 & 29.7 & 33.0 & 36.3 \\
\hline $\begin{array}{l}\text { Some secondary } \\
\text { education }\end{array}$ & 19.0 & 33.7 & 55.5 & 14.7 & 16.3 & 7.7 & 28.7 & 48.2 & 623 \\
\hline $\begin{array}{l}\text { Low household } \\
\text { amenities }\end{array}$ & 9.3 & 7.0 & 32.4 & 25.2 & 21.5 & 13.7 & 28.2 & 27.7 & 41.6 \\
\hline $\begin{array}{l}\text { Medium } \mathrm{HH} \\
\text { amenities }\end{array}$ & 14.5 & 13.6 & 43.7 & 22.8 & 20.4 & 11.2 & 28.0 & 33.5 & 47.8 \\
\hline $\begin{array}{l}\text { High houschold } \\
\text { amenities }\end{array}$ & 18.0 & 27.1 & 55.9 & 18.5 & 17.4 & 6.2 & 26.2 & 41.1 & 45.6 \\
\hline
\end{tabular}

Excluding women who were pregnant or had never had sex

'The score is created by combining the significant women's socio-economic factors and urban-rural residence. For binary variables, the factor representing the lowest contraceptive use was coded as zero and the category with the bighest use as one. For other variables, the intermediate calegories were coded by dividing their beta coefficient by the bighest beta coefficient to give a value between zero and one. The recoded variables were then summed to create the score, which was categorised and tested in the model. 


\section{Table 2 Study Population}

\begin{tabular}{lrrr}
\hline & Ghana & Tanzania & Zimbabwe \\
\hline Number of primary sampling units & 400 & 357 & 230 \\
Number of women interviewed & 4,843 & 8,120 & 6,128 \\
Number of women 'at risk' of conception & 3,784 & 6,296 & 4,402 \\
\hline
\end{tabular}




\section{Table 5 Odds Ratios of Modern Contraceptive Use in Three Selected African Countries}

\begin{tabular}{|c|c|c|c|}
\hline Factor & Ghana & Tanzania & Zimbabwe \\
\hline \multicolumn{4}{|l|}{ Wants amotber cbild in next 12 montbs } \\
\hline Yes & 1.00 & 1.00 & 1.00 \\
\hline Another answer & $4.44 \cdots$ & $4.60 * * *$ & $12.74^{\prime \prime \prime}$ \\
\hline \multicolumn{4}{|l|}{ Age (jears) } \\
\hline $15-19$ & 1.04 & 0.61 & 1.44 \\
\hline $20-29$ & 0.97 & 0.97 & $1.67^{* \cdots}$ \\
\hline $30-39$ & 1.00 & 1.00 & 1.00 \\
\hline $40-44$ & 0.78 & 1.20 & $0.57^{\cdots \cdots}$ \\
\hline $45-49$ & $0.44^{* \cdots}$ & 0.83 & $0.23 * *$ \\
\hline \multicolumn{4}{|l|}{ No. of living cbildren } \\
\hline None & 1.00 & 1.00 & 1.00 \\
\hline One & 0.73 & $2.03 * \cdots$ & $3.64 k 0$ \\
\hline Tivo & 0.59 * & $2.75 * * *$ & $5.41^{* * *}$ \\
\hline Three or more & 1.21 & $3.84 * \cdots$ & $4.96^{\times 12}$ \\
\hline \multicolumn{4}{|l|}{ Marifal status } \\
\hline Never marricd & $3.06 \cdots$ & $2.08 *$ & $1.74^{*}$ \\
\hline Monogamous unions & $266 * \cdots$ & 1.03 & $3.33^{\mathrm{mot}}$ \\
\hline Polygynous unions & $1.99^{*}$ & 0.77 & $2.00^{* \cdots}$ \\
\hline Formerly marricd & 1.00 & 1.00 & 1.00 \\
\hline \multicolumn{4}{|l|}{ Religion } \\
\hline Catholic & 1.00 & 1.00 & - \\
\hline Protestant & 0.85 & $1.56^{* *}$ & - \\
\hline Muslim & 0.78 & $1.31^{\circ}$ & - \\
\hline Traditional/None/Other & $0.53^{*}$ & $0.23 *$ & $0.73^{* \cdots}$ \\
\hline Christian & - & - & 1.00 \\
\hline \multicolumn{4}{|l|}{ Place of residence } \\
\hline Rural & & 1.00 & 1.00 \\
\hline Urban & & $.78 * \cdots$ & $1.16^{\circ}$ \\
\hline \multicolumn{4}{|l|}{ Educational leovl } \\
\hline No education & & 1.00 & \\
\hline Primary incomplete & & $1.7 * *$ & \\
\hline Complete primary & & $2.18^{* * *}$ & \\
\hline Some second/higher & & $2.67 * n$ & \\
\hline \multicolumn{4}{|l|}{ Occupation } \\
\hline Not working & & & 1.00 \\
\hline Skilled manual & & & 1.17 \\
\hline Agriculture - self-cmployed & & & $1.32^{*}$ \\
\hline Clerical/services & & & $1.53 \cdots$ \\
\hline Prof./tech./manag./sales & & & $2.69 * * *$ \\
\hline Unskilled manual & & & $3.27^{* * *}$ \\
\hline \multicolumn{4}{|l|}{ Housebold amenities score } \\
\hline Low & 1.00 & 1.00 & 1.00 \\
\hline Mcdium & $1.64^{*+*}$ & $1.48 *$ & $1.47^{\cdots}$ \\
\hline Iligh & $1.90 * 0$ & $1.87 * \cdots$ & $1.91^{* * * *}$ \\
\hline \multicolumn{4}{|l|}{ Parfner's educational level } \\
\hline No culucation & 1.00 & 1.00 & 1.00 \\
\hline Primary incomplete & $1.92^{*}$ & 1.03 & 1.28 \\
\hline Complete primary & $214^{* * *}$ & $1.71^{* * *}$ & 1.34 \\
\hline Some secondary/higher & $217^{* * * k}$ & $1.77^{* *}$ & $1.78 * a t$ \\
\hline Unknown & 1.27 & - & - \\
\hline
\end{tabular}


http://www.bioline.org.br/showimage?rh/photo/rh04034t5.jpg

some sccondary/ migner

Unknown

Partwer's occupation

Agricultural sclf-cmployed/not working

Other cmplovment

Odds ralio prediating curremt use of a modern contraceplive method; Region also induded in eadb model ${ }^{*} p<0.05 ;{ }^{* *} p<0.01 ;{ }^{* *} p<0.001 ; n=3750$ (Ghama); $n=6001$ (Tawzania); $n=4267$ (Zimbalnve) - denotes factor not included in tbe model 